\title{
Free dietary advice via the Internet as a tool for prevention and treatment of chronic non-communicable diseases - preliminary reports
}

\author{
Magdalena Siuba-Strzelińska, Klaudia Wiśniewska and Mirosław Jarosz \\ Institute of Food and Nutrition, Warszawa, Poland
}

\section{Abstract}

Introduction: Currently, there are no dietary consultations in the health service in Poland, that is why free access to the dietician is often difficult. The use of modern teleinformation technologies in medicine, creates unprecedented possibilities of providing medical services. Therefore, as part of the National Health Program financed by the Ministry of Health, the first digital platform was established in Poland - the Online Diet Center, where everyone with access to the Internet can use the advice of a professional dietitian free of charge.

The aim of the study was to obtain information on the characteristics of patients reporting online consultations and identifying their needs.

Materials and methods: The study was conducted among the patients of the Online Diet Center during the 12 months of running a diet counseling (December 2017-December 2018) using the authorial questionnaire that each patient completed after creating an account on the online counseling platform before and after the advice (satisfaction survey). Each advice lasted 30 minutes and was conducted by a trained dietitian.

Results: The number of patients who created an account on the Dietetic Online Center platform was 10,850, of which $23 \%$ were men and $77 \%$ women. Most often it was used by residents of large cities (including Warsaw, Krakow, Katowice, Wrocław, Poznań, Gdańsk, Łódź). Almost 2\% of patients were also Poles living abroad (Denmark, Australia, Great Britain, Ireland, Germany, Norway, Belgium, Italy, Spain, Brazil). Almost $1 / 3$ of all registered patients after one consultation had another consultation with a dietitian. The most common disease was obesity (17\%). Patients also declare hypothyroidism (15\%), hypertension (13\%), food allergy or intolerance $(6.8 \%)$, eating disorders $(5.6 \%)$ and depression $(5 \%)$. Only $10 \%$ of patients were people who did not report any diseases. Only less than $1 \%$ of patients were not satisfied with such a consultation system, explaining that the information provided by the dietician was not useful. The duration of the advice was sufficient for patients.

Discussion: A large group of patients who apply to the Online Diet Center were women, which is consistent with the general trend in the area of dietary services. Due to the low frequency of using online dietary advice in small towns, in year 2019 a promotional campaign will be addressed to these places. The low percentage of dissatisfied patients and the willingness to make further advices, testifies to the success of platform of online dietary counseling and the legitimacy of its further development.

\section{Conflict of Interest}

There is no conflict of interest 